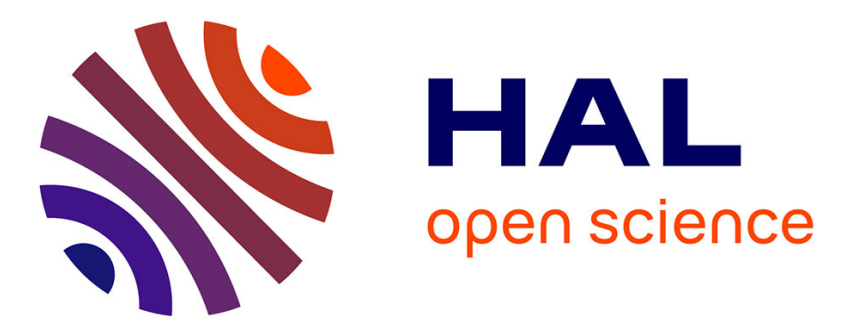

\title{
Robustness of a multimodal piezoelectric damping involving the electrical analogue of a plate
}

Boris Lossouarn, Kenneth A. Cunefare, Mathieu Aucejo, Jean-François Deü

\section{To cite this version:}

Boris Lossouarn, Kenneth A. Cunefare, Mathieu Aucejo, Jean-François Deü. Robustness of a multimodal piezoelectric damping involving the electrical analogue of a plate. SPIE Smart Structures and Materials + Nondestructive Evaluation and Health Monitoring, Apr 2016, Las Vegas, United States. 10.1117/12.2218357 . hal-01739219

\section{HAL Id: hal-01739219 \\ https://hal.science/hal-01739219}

Submitted on 21 Mar 2018

HAL is a multi-disciplinary open access archive for the deposit and dissemination of scientific research documents, whether they are published or not. The documents may come from teaching and research institutions in France or abroad, or from public or private research centers.
L'archive ouverte pluridisciplinaire HAL, est destinée au dépôt et à la diffusion de documents scientifiques de niveau recherche, publiés ou non, émanant des établissements d'enseignement et de recherche français ou étrangers, des laboratoires publics ou privés. 


\title{
Robustness of a multimodal piezoelectric damping involving the electrical analogue of a plate
}

\author{
Boris Lossouarn ${ }^{\mathrm{a}}$, Kenneth A. Cunefare ${ }^{\mathrm{b}}$, Mathieu Aucejo ${ }^{\mathrm{a}}$, and Jean-François Deü ${ }^{\mathrm{a}}$ \\ ${ }^{a}$ Conservatoire National des Arts et Métiers, 2 Rue Conté, Paris, France \\ ${ }^{\mathrm{b}}$ Georgia Institute of Technology, 813 Ferst Dr, Atlanta, USA
}

\begin{abstract}
Multimodal passive damping of a mechanical structure can be implemented by a coupling to a secondary structure exhibiting similar modal properties. When considering a piezoelectric coupling, the secondary structure is an electrical network. A suitable topology for such a network can be obtained by a finite difference formulation of the mechanical equations, followed by a direct electromechanical analogy. This procedure is applied to the Kirchhoff-Love theory in order to find the electrical analogue of a clamped plate. The passive electrical network is implemented with inductors, transformers and the inherent capacitance of the piezoelectric patches. The electrical resonances are tuned to approach those of several mechanical modes simultaneously. This yields a broadband reduction of the plate vibrations through the array of interconnected piezoelectric patches. The robustness of the control strategy is evaluated by introducing perturbations in the mechanical or electrical designs. A non-optimal tuning is considered by way of a uniform variation of the network inductance. Then, the effect of local or boundary modifications of the electromechanical system is observed experimentally. In the end, the use of an analogous electrical network appears as an efficient and robust solution for the multimodal control of a plate.
\end{abstract}

Keywords: vibration control, multimodal coupling, passive damping, piezoelectric network, robustness

\section{INTRODUCTION}

Control solutions involving an array of piezoelectric resonant shunts have been proposed in order to damp vibration of plates. ${ }^{1-3}$ The use of several piezoelectric patches of reduced dimensions limits the problem of charge cancellation and can eventually introduce an additional stop band effect over the considered frequency range. However, this strategy does not apply in the low frequency range, where the simultaneous control of several plate modes requires the synthesis of multiresonant shunts. Such shunts with multiple inductors were proposed by $\mathrm{Wu}^{4}$ and then implemented on a one-dimensional piezoelectric array. ${ }^{5}$ The multimodal approach of $\mathrm{Wu}$ and other similar concepts require $n$ inductors per patch to affect $n$ modes, which may lead to a system with an impractically large number of components. A broadband control can still be implemented with negative capacitance, which simplifies the electrical layout and offers good performance. ${ }^{6-8}$ The main drawback is that a negative capacitance needs to be synthesized with an active circuit and the control can thus suffer from stability issues. A solution for the multimodal control of a plate with a passive electrical network was presented by Vidoli and dell'Isola. ${ }^{9}$ The network consists of distributed inductors that interconnect the piezoelectric patches in order to synthesize electrical modes that match the mechanical modes, both in the spatial and frequency domains. This idea was then extended by Giorgio et al. ${ }^{10}$ to systems involving only a few piezoelectric patches. The main limit remains the practical implementation of a suitable passive network. As we need to reproduce the modal properties of mechanical structures with an electrical system, suitable topologies can be found by applying an electromechanical analogy. ${ }^{11,12}$ In 1949, MacNeal ${ }^{13}$ already proposed and validated electrical analogues for numerous mechanical structures as rods, beams and plates. Those passive networks finally reappeared in

Further author information: (Send correspondence to B.L.)

B.L.: E-mail: boris.lossouarn@cnam.fr, Telephone: +33140272328

K.A.C.: E-mail: ken.cunefare@me.gatech.edu, Telephone: +1 4048944726

M.A.: E-mail: mathieu.aucejo@cnam.fr, Telephone: +33158 808581

J.F.D.: E-mail: jean-francois.deu@cnam.fr, Telephone: +331 40272760 
the analogous control of one-dimensional structures. ${ }^{14-17}$ With this approach, the number of components per piezoelectric patch is independent of the number of modes that are targeted. For the control of thin plates, Alessandroni et al. ${ }^{18}$ proposed an analogous network ensuring a broadband piezoelectric damping. Unfortunately, the large number of electrical components makes difficult its practical implementation.

A simpler electrical network can be obtained by reconsidering the Kirchhoff-Love theory. The new topology is presented in the present paper and applied to the damping of a clamped plate. It thus becomes possible to evaluate the robustness of the control strategy with respect to a real experimental setup. The effect of parameter variations needs to be investigated because it was shown that solutions involving resonant shunts can be very sensitive to electrical mistunings. ${ }^{19,20}$ In practical applications, even if the robustness can be slightly improved with an overestimated damping, ${ }^{21}$ it seems difficult to maintain a fine tuning of a single resonant shunt without autonomous adaptation of the electrical parameters. ${ }^{22}$ Nevertheless, in the case of a distributed solution involving an array of resonant shunts, uncertainties between components does not lead to a significant degradation of the performance. ${ }^{23}$ This is also true with electrical analogous networks as it was shown that experiments involving inductors with a $\pm 10 \%$ tolerance were in good agreement with deterministic simulations. ${ }^{14}$ Then, the question related to the effect of an uniform detuning of the electrical parameters still arises, because it could seriously affect the damping performances. ${ }^{24}$ Another issue concerns the influence of defects appearing locally or along the boundary of the mechanical or the electrical domains.

In this study, a discrete model of a thin plate is proposed in order to define its analogous electrical network. This network is implemented experimentally and then coupled to a clamped plate through an array of piezoelectric patches. The observed broadband vibration reduction validates the damping strategy and gives a reference for the evaluation of its robustness with respect to parameter variations. In a second part, the performance loss induced by an uniform variation of the network inductance is quantified. Then, local or boundary modifications are introduced experimentally. The mechanical properties are altered by adding a lumped mass on the plate or by removing some of the bolts ensuring the clamping condition. The electrical network is also modified by doubling an inductance or short-circuiting a line of piezoelectric patches.

\section{MULTIMODAL CONTROL BY ANALOGOUS COUPLING}

A finite difference method is applied to the Kirchhoff-Love plate theory in order to get a discrete mechanical model. This model is then converted in the electrical domain by applying a direct electromechanical analogy. The analogous electrical network is implemented and coupled to a clamped plate in order to damp structural vibrations.

\subsection{Finite difference model of a plate}

According to the Kirchhoff-Love theory, the governing differential equation for a thin plate of Young's modulus $Y$, density $\rho$, Poisson's ratio $\nu$ and thickness $h$ is given by

$$
-D\left(\frac{\partial^{4} w}{\partial x^{4}}+2 \frac{\partial^{4} w}{\partial x^{2} \partial y^{2}}+\frac{\partial^{4} w}{\partial y^{4}}\right)=\rho h \frac{\partial^{2} w}{\partial t^{2}}, \quad \text { where } \quad D=\frac{Y h^{3}}{12\left(1-\nu^{2}\right)} .
$$

$w$ represents the transverse displacement, $x$ and $y$ are the two space variables, $t$ is the time variable and $D$ is the bending stiffness. The angles along the principal directions satisfy the Kirchhoff-Love assumption which states that the normals to the undeformed midplane remains straight after deformation: $\theta_{x}=\frac{\partial w}{\partial x}$ and $\theta_{y}=\frac{\partial w}{\partial y}$. Consequently, the system of equations

$$
\begin{aligned}
& \frac{\partial Q_{x}}{\partial x}+\frac{\partial Q_{y}}{\partial y}=\rho h a \frac{\partial^{2} w}{\partial t^{2}} \\
& M=a D\left(\frac{\partial \theta_{x}}{\partial x}+\frac{\partial \theta_{y}}{\partial y}\right)
\end{aligned}
$$

is equivalent to Eq. (1) when the shear forces are defined by $Q_{x}=-\frac{\partial M}{\partial x}$ and $Q_{y}=-\frac{\partial M}{\partial y}$. The constant $a$ that appears in Eq. (2) corresponds to the side of a square plate unit cell. This continuous plate element is 


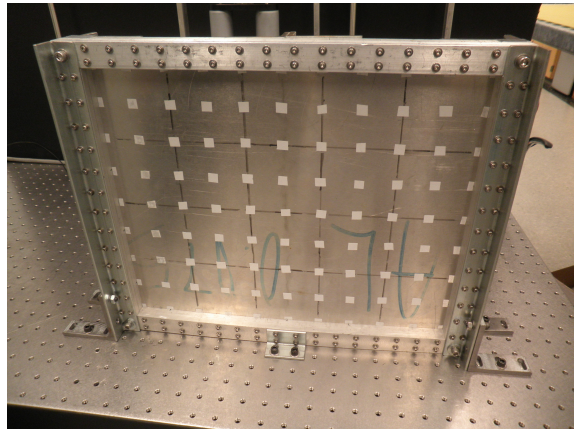

(a)

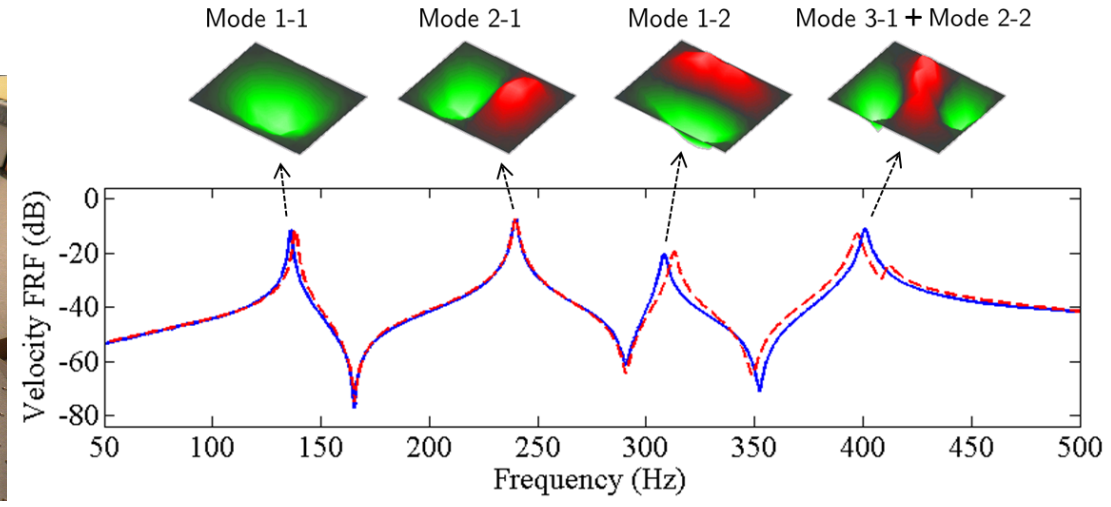

(b)

Figure 1: Experimental validation of the finite difference model: (a) Clamped plate. (b) Experimental operating deflection shapes with respect to the velocity FRF - (-) experimental FRF, (--) simulated FRF computed with $15 \times 12$ unit cells.

discretized by applying the following finite difference scheme: $\frac{\partial[.]}{\partial x} \approx \frac{[\cdot]_{R}-[.]_{L}}{a}$ and $\frac{\partial[.]}{\partial y} \approx \frac{[\cdot]_{T}-[.]_{B}}{a}$, where $L$, $R, B$ and $T$ refer respectively to the left, right, bottom and top sides of the square unit cell. This central finite difference approximation is applied to Eq. (2), which gives

$$
\begin{aligned}
& -Q_{L}+Q_{R}-Q_{B}+Q_{T}=m \ddot{w}_{I} \\
& -M_{I}=D\left(\theta_{L}-\theta_{R}+\theta_{B}-\theta_{T}\right)
\end{aligned},
$$

where $I$ refers to the position at the center of the unit cell and $m=\rho h a^{2}$ is the mass of the unit cell. Note that that $w_{I}$ and $M_{I}$ are related to the side variables thanks to forward or backward finite differences. For example, it can be written that $w_{I}=w_{L}+\frac{a}{2} \theta_{L}$ and $M_{I}=M_{L}-\frac{a}{2} Q_{L}$. In the end, the dynamic stiffness matrix of a discrete unit cell can be defined from the relation between the displacement vector $\left[w_{B} ; \theta_{B} ; w_{L} ; \theta_{L} ; w_{R} ; \theta_{R} ; w_{T} ; \theta_{T}\right]$ and the force vector $\left[Q_{B} ; M_{B} ; Q_{L} ; M_{L} ; Q_{R} ; M_{R} ; Q_{T} ; M_{T}\right]$. A classical assembly process leads to the global dynamic stiffness matrix of a plate made of several square unit cells. It becomes then possible to compute the dynamic behavior of a plate as long as the length of the chosen unit cells is small compared to the considered wavelength.

The finite difference model is validated experimentally by considering the clamped aluminum plate in Fig. 1(a), which is $400 \mathrm{~mm}$ long, $320 \mathrm{~mm}$ wide and $1.9 \mathrm{~mm}$ thick. A shaker applies a white noise excitation on one side of the plate and the input is measured with a force sensor. A scanning laser vibrometer gives access to the velocity field on the other side of the plate. The velocity frequency response functions (FRFs) are then measured over a frequency range spanning from $50 \mathrm{~Hz}$ to $500 \mathrm{~Hz}$. Readers can refer to Lossouarn et al. ${ }^{25}$ for more details about the experimental setup. As seen in Fig. 1(b), four resonances are observed over the considered frequency range. Actually, the $4^{\text {th }}$ resonance is a combination of the $4^{\text {th }}$ and $5^{\text {th }}$ modes that exhibit close natural frequencies. The finite difference model is implemented with $15 \times 12$ unit cells in order to ensure more than 10 unit cells per wavelength for the first five plate modes. It is observed in Fig. 1(b) that the numerical results are in good agreement with the experimental ones. The main difference remains in the fact that the $4^{\text {th }}$ and $5^{\text {th }}$ modes can be distinguished on the simulated FRF. It is still remarked that refining the mesh does not clearly improve the correlation because it can not overcome the bias introduced by the non-ideal clamped boundary conditions in the experiments.

\subsection{Analogous electrical network}

Once the proposed finite difference model is validated, it can be converted into its analogous network made of discrete electrical components. Contrary to previous studies on plate electrical analogues, ${ }^{13,18}$ we focus on the direct electromechanical analogy, which is compatible with the electrical representation of a piezoelectric 


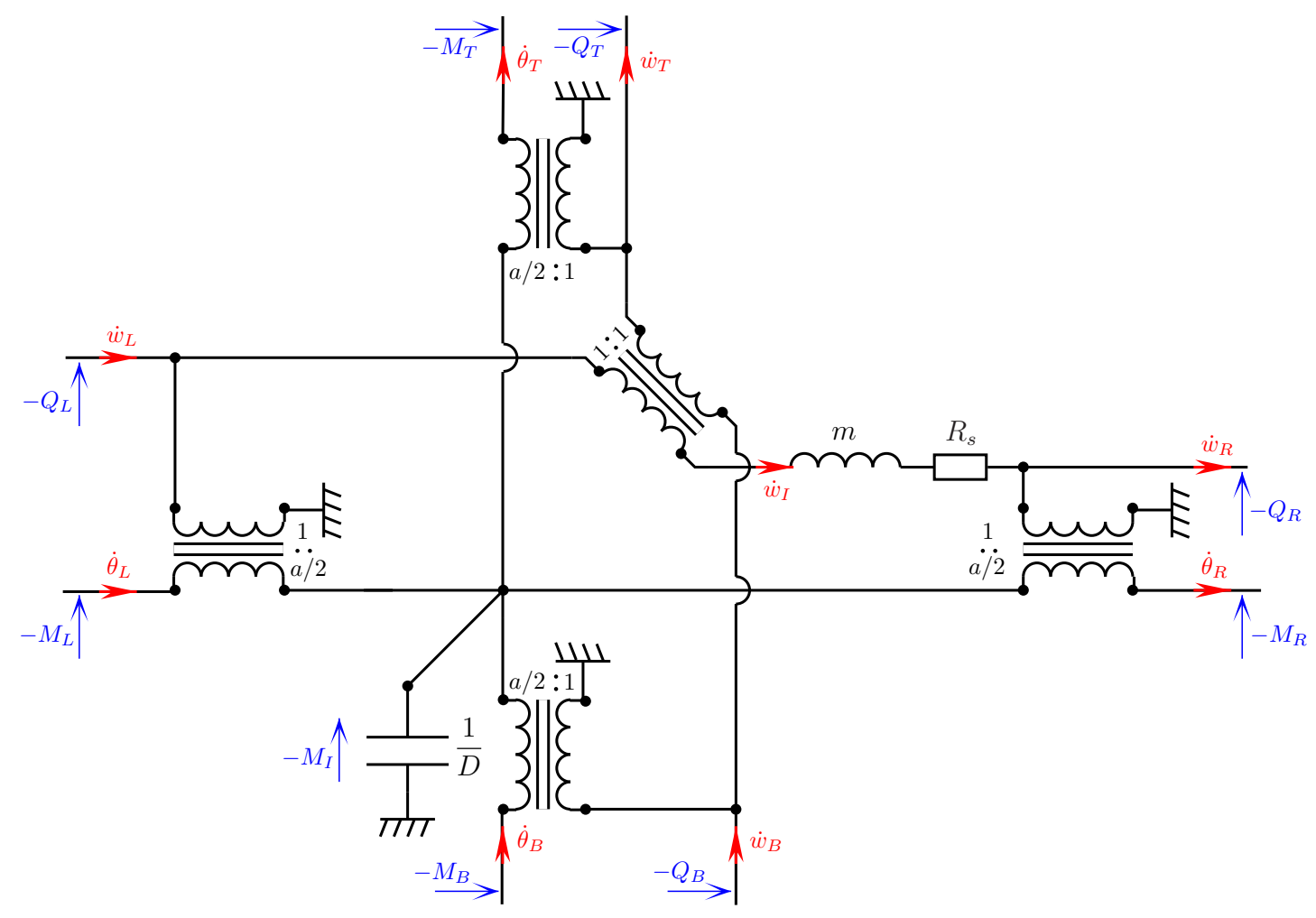

Figure 2: Electrical unit cell as a direct analogue of the finite difference model of a plate unit cell; $R_{s}$ represents additional damping, $R_{s}=0$ when no damping is considered.

transducer. ${ }^{11}$ This direct analogy is based on the velocity-current and force-voltage equivalences. It then becomes possible to represent the system of equations (3) with the electrical scheme in Fig. 2. The electrical transformers of ratio $a / 2$ allow the implementation of the forward and backward finite differences that satisfy the definition of $\theta$ and $Q$ as spatial derivatives of $w$ and $-M$, respectively. According to the direct electromechanical analogy, the inductance represents the mass of the unit cell and the capacitance is equal to the inverse of the bending stiffness. The proposed two-dimensional unit cell in Fig. 2 can finally be seen as an extension of the direct analogue of an Euler-Bernouilli beam. ${ }^{13,15-17}$

The electrical analogue of a plate is built by connecting together $5 \times 4$ identical unit cells. This coarse mesh was chosen for practical reasons related to the number of electrical components. It can obviously not implement a minimum of 10 unit cells per wavelength up to the $5^{\text {th }}$ mode, which was suggested in Sec. 2.1. Consequently, there is no reason to expect an accurate correlation between the mechanical and electrical resonance frequencies but we can still expect distributions of the electrical current that properly reproduce plate-like mode shapes. As we focus on the analogue of a clamped plate, the zero-displacement and zero-angle boundary conditions are implemented by keeping the electrical ports open at the boundaries. Electrical simplifications occurs and the network is finally assembled with 16 capacitors $C=470 \mathrm{nF}, 17$ transformers of ratio 1:4, 6 transformers of ratio 1:1 and 6 inductors $L=0.9 \mathrm{H}$. The internal resistance of the transformers is evaluated and integrated in the electrical scheme of Fig. 2. Another element to consider is the resistance $R_{s}$ that can be added in series with the inductors $L$ for a tuning purpose. It is then possible to properly simulate the periodic electrical network presented in Fig. 3(a). As an example, it is shown in Fig. 3(b) that the addition of serial resistors $R_{s}=180 \Omega$ gives an electrical FRF, where the 6 electrical resonances induced by the 6 inductors are hardly observable. On the other hand, the resonances are clearly identified when $R_{s}=0 \Omega$. They can still be qualified as smooth when compared to the sharp resonances of a lightly damped mechanical system as the plate presented in Fig. 1. The relatively low quality factor of the electrical resonances is mainly due to the internal resistance of the transformers. In Fig. 3(b), it is observed that the simulation fits with the FRF obtained experimentally. Moreover, the spatial distributions 


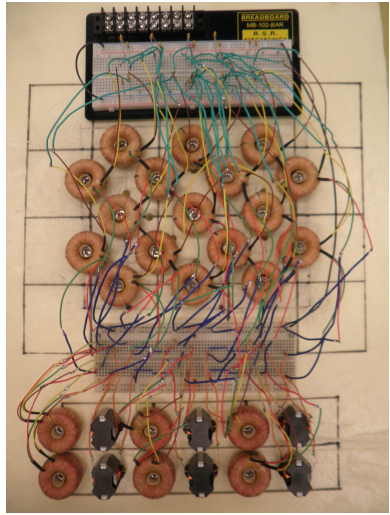

(a)

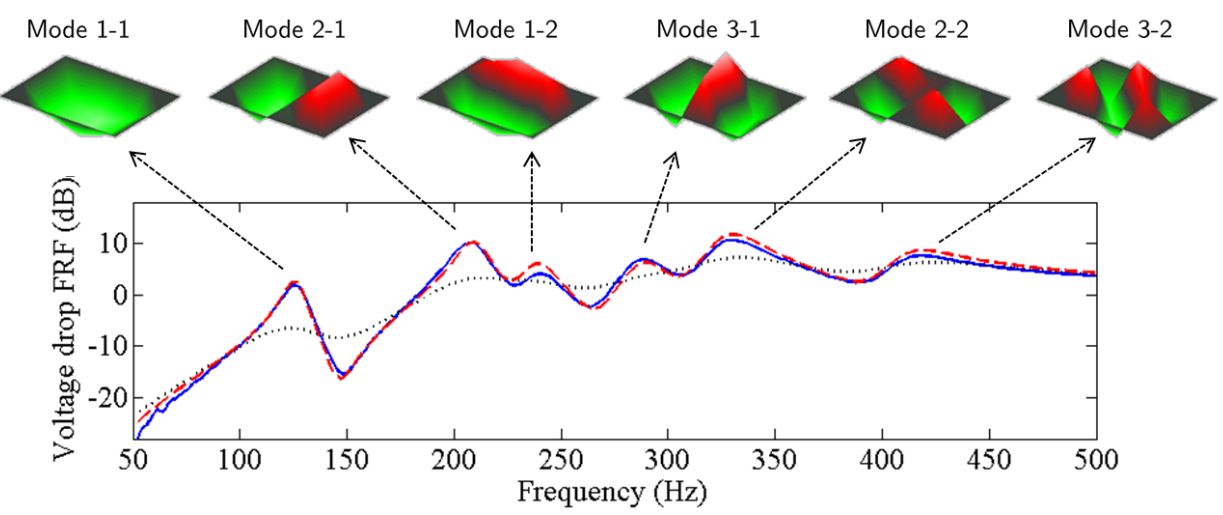

(b)

Figure 3: Experimental validation of the analogous network: (a) Electrical network implemented with $5 \times 4$ unit cells. (b) Experimental current distributions with respect to the voltage FRF with $L=0.9 \mathrm{H}$ and $C=$ $470 \mathrm{nF}$ - (-) experimental FRF with $R_{s}=0 \Omega,(--)$ simulated FRF with $R_{s}=0 \Omega,(\cdots)$ simulated FRF with $R_{s}=180 \Omega$.

of the currents around the resonances approximates the mode shapes of a clamped plate. This validates the analogue network topology and confirms that the proposed finite difference scheme is able to reproduce the dynamic behavior of a plate.

\subsection{Multimodal coupling through piezoelectric patches}

An electrical network approximating the modal properties of a plate is implemented and it can synthesize an efficient broadband controller. Indeed, it was shown that the piezoelectric coupling of a mechanical structure to its electrical analogue leads to a passive control that acts as a multimodal tuned mass damping. ${ }^{14-17}$ The exact analogue of a continuous structure can obviously not be obtained with a set of discrete electrical components. Nevertheless, a discrete network can still approximate the mechanical modes and interact with them in order to damp the structural response. ${ }^{14,15,17}$ This is illustrated in a two-dimensional case by coupling the clamped aluminum plate to the electrical network made of $5 \times 4$ unit cells. As shown in Fig. 4(a), an array of 20 square PZT-5H patches of side $72.4 \mathrm{~mm}$ and $0.27 \mathrm{~mm}$ thickness cover most of the plate surface. Those piezoelectric

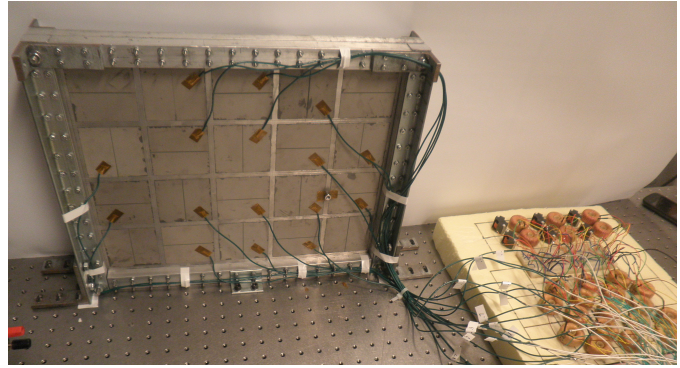

(a)

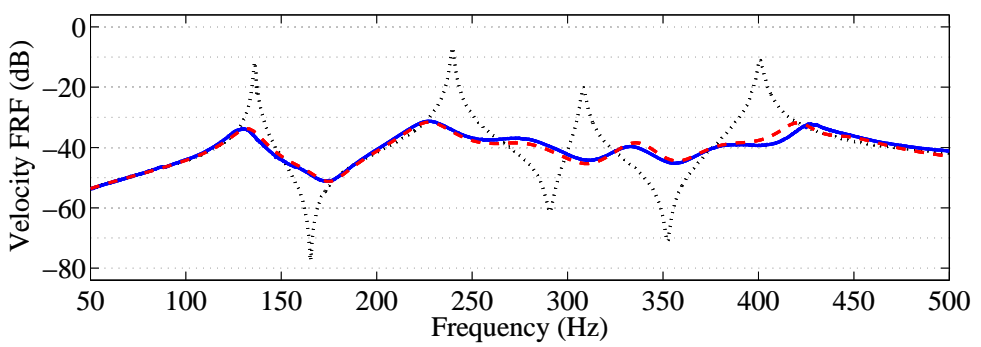

(b)

Figure 4: Coupling of the plate to its analogous network: (a) Clamped plate coupled to the electrical network through an array of $5 \times 4$ piezoelectric patches. (b) Effect of the multimodal control on the velocity FRF of the plate - $(\cdots)$ experimental FRF with short-circuited patches, $(-)$ experimental FRF with the analogous network tuned to $L=0.7 \mathrm{H}$ and $R_{s}=180 \Omega,(--)$ simulated FRF with the analogous network tuned to $L=0.7 \mathrm{H}$ and $R_{s}=180 \Omega$. 
patches are connected to the electrical network by assuming the role of the capacitors. The effect of the coupling is observed in Fig. 4(b) with $L=0.7 \mathrm{H}$ and $R_{s}=180 \Omega$ resistors added in series with the six inductors. Those values were obtained from a $\mathcal{H}_{\infty}$ optimization, which minimizes the maximum of the velocity FRF over the 50 $\mathrm{Hz}$ to $500 \mathrm{~Hz}$ frequency range. The vibration reduction is above $20 \mathrm{~dB}$ for the first 5 modes of the plate. This shows that the strategy offers an efficient control, even with a coarse discretization of the electrical network.

\section{ROBUSTNESS OF THE CONTROL STRATEGY}

The robustness of the damping solution is evaluated by introducing a uniform variation of the electrical components. Then, local defects are added experimentally in order to observe the effect on control performance. For the same purpose, an alteration of the electrical or mechanical boundary conditions is finally considered.

\subsection{Uniform detuning of the electrical network}

A first observation related to the robustness of the strategy can be introduced by considering the difference between the natural frequencies of the plate and those of the electrical controller. Recall that the analogue network is discrete, so it can not perfectly match several mechanical resonances simultaneously. The natural frequencies are compared in Table 1 when $L$ is set to $0.7 \mathrm{H}$. It is seen that the $\mathcal{H}_{\infty}$ optimization over the $50 \mathrm{~Hz}$ to $500 \mathrm{~Hz}$ frequency range gives a positioning of the electrical natural frequencies that differs up to $18 \%$ from the mechanical ones. Thus, an imprecise tuning of an electrical resonance can still lead to a $20 \mathrm{~dB}$ reduction of its mechanical counterpart, as observed in Fig. 4(b). This can be explained by the fact that the optimal electrical network is highly dissipative. Indeed, it is seen in Fig. 3(b) that a resistance $R_{s}=180 \Omega$ induces a relatively flat electrical FRF. This makes the control more robust, as it becomes less sensitive to electrical tuning variations.

The performance loss due to a detuning of the electrical parameters is evaluated by introducing a variation of the inductance $L$ around its optimal value. A variation of the piezoelectric capacitance could also be analyzed but it would lead to similar conclusions. Furthermore, a variation of the resistance $R_{s}$ is not considered because it is observed that its influence is relatively low, which extends results obtained for a single resonant shunt. ${ }^{5,19}$ A $\pm 30 \%$ uniform variation of $L$ is considered, so that the electrical unit cells have a same inductance all over the network, but this inductance varies between $0.49 \mathrm{H}$ and $0.91 \mathrm{H}$. This range is introduced in the electromechanical model that was validated in Fig. 4(b). By extracting the highest and lowest amplitude computed at each frequency point, we get the simulated frequency response envelope presented in Fig. 5. The optimal FRF should always stay inside the frequency response envelope because its inductance corresponds to the center of the range of

Table 1: Comparison of mechanical and electrical natural frequencies with $L=0.7 \mathrm{H}$.

\begin{tabular}{|l|l|l|l|l|l|}
\hline & Mode 1-1 & Mode 2-1 & Mode 1-2 & Mode 3-1 & Mode 2-2 \\
\hline Clamped plate & $138 \mathrm{~Hz}$ & $242 \mathrm{~Hz}$ & $315 \mathrm{~Hz}$ & $409 \mathrm{~Hz}$ & $411 \mathrm{~Hz}$ \\
\hline Electrical network & $163 \mathrm{~Hz}$ & $270 \mathrm{~Hz}$ & $309 \mathrm{~Hz}$ & $374 \mathrm{~Hz}$ & $424 \mathrm{~Hz}$ \\
\hline
\end{tabular}

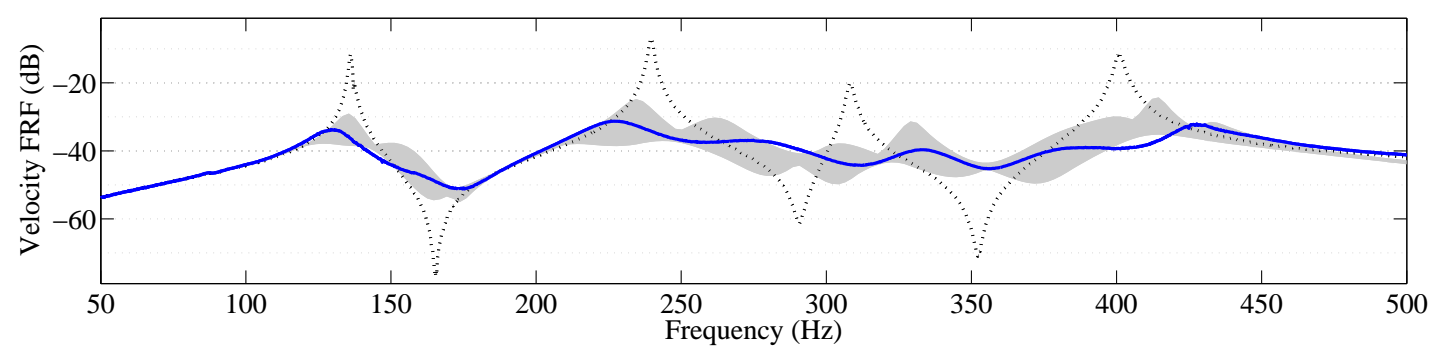

Figure 5: Sensitivity of the velocity FRF with respect to an inductance variation $-(\cdots)$ experimental FRF with short-circuited patches, $(-)$ experimental FRF with the analogous network tuned to $L=0.7 \mathrm{H}$ and $R_{s}=180 \Omega$, $(\square)$ simulated frequency response envelope with a $\pm 30 \%$ variation on $L$. 


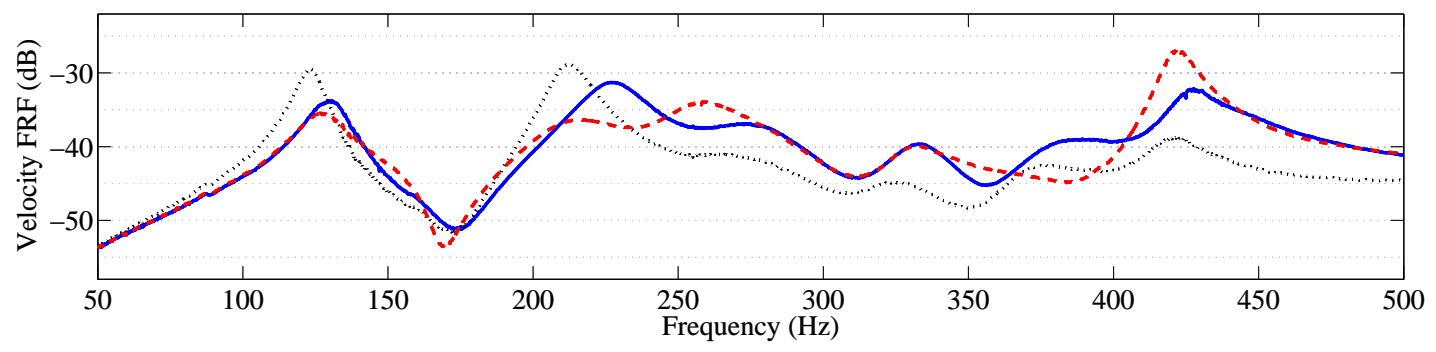

Figure 6: Influence of local defects - (-) optimal FRF with $L=0.7 \mathrm{H}$ and $R_{s}=180 \Omega,(\cdots)$ with a doubled mass in one mechanical unit cell, $(--)$ with a doubled inductance in one electrical unit cell.

variation. The fact that the experimental FRF can sometimes leave the envelope in Fig. 5 is simply due to slight deviations of the model, according to what is observed in Fig. 4(b). In any case, a $\pm 30 \%$ variation on $L$ induces a loss of $7 \mathrm{~dB}$ on the min-max criterion related to the $\mathcal{H}_{\infty}$ optimization. This loss of performance is non-negligible but it still maintains a vibration reduction above $85 \%$ with respect to the maximum amplitude of the uncontrolled velocity FRF.

\subsection{Local defect in a mechanical or electrical unit cell}

Local modifications of the electromechanical system can also affect the control performance. For example, a local damage can appear on the mechanical structure, which would modify the mass or the stiffness of one specific unit cell. On the other hand, the degradation of an electrical component might alter the modal properties of the entire network. A first experiment considers a lumped mass addition of 50 gram on the clamped plate, so that the mass of one of the twenty $8 \times 8 \mathrm{~cm}^{2}$ mechanical unit cell is doubled. This added mass moves the plate resonances to lower frequencies $\left(-5 \%\right.$ for the $1^{\text {st }}$ resonance, $-8 \%$ for the $2^{\text {nd }}$ resonance), which detunes the control strategy. Considering mode 1-1 and mode 2-1 in Table 1, the gap between the mechanical and electrical frequencies was already significant before the addition of a local defect. After modification, the gap is even larger, which accounts for the $3 \mathrm{~dB}$ loss that appears below $250 \mathrm{~Hz}$ in Fig. 6. On the other hand, we note that the local mass addition is beneficial above $250 \mathrm{~Hz}$ because it better tunes the resonance distribution for the highest modes.

In the electrical domain, the equivalent of a mass addition is an inductance increase on the same unit cell. Thus, one inductance of the network is doubled to implement the counterpart of the previous experiment. This local defect moves the electrical resonances to lower frequencies. Consequently, the last FRF shown in Fig. 6 evolves in opposition to what was observed after the modification of the mechanical structure. Indeed, the tuning of the electrical network is here improved for the modes 1-1 and 2-1 but the performance is decreased above 250 Hz. The performance loss is mainly due to mode $3-1$, which exhibits a $5 \mathrm{~dB}$ increase of the velocity around 420 $\mathrm{Hz}$.

\subsection{Modifications of the boundary conditions}

A second set of experiments focuses on modifications of the mechanical or electrical boundary conditions. An alteration of plate boundary conditions is firstly considered by removing the bolts all along the upper bar of the clamping frame. So, the rotations and transverse displacements are no longer blocked on the upper side of the plate. This modifies the mechanical modes, especially their distribution over the frequency range. The stiffness reduction shifts the resonances to lower frequencies, as with the mass addition. Consequently, it is observed in Fig. 7 similar results to what was presented Fig. 6 when looking at the performance loss around the first two modes. Note a $3 \mathrm{~dB}$ loss compared to the optimal FRF. The analysis above $250 \mathrm{~Hz}$ is less obvious because of the strong modification of the mechanical modal distribution. In any case, we remark that a significant alteration of the mechanical boundary conditions does not defeat the control strategy.

The electrical equivalent of the stiffness reduction along the upper boundary is approximated by shortcircuiting the upper line of piezoelectric patches. Similarly to what appears after an increase of the inductance, the electrical resonances move to lower frequencies. This better tunes the control of the first two modes as seen in 


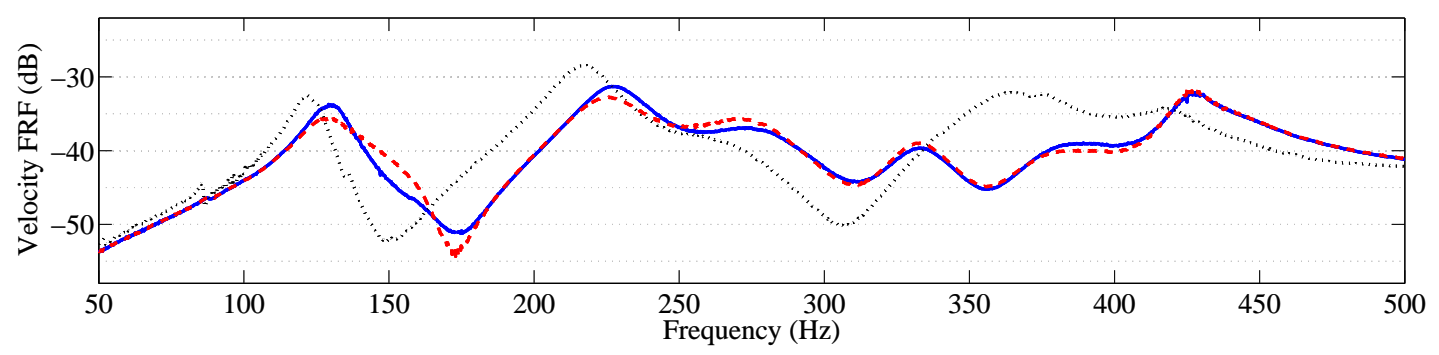

Figure 7: Influence of boundary conditions - (-) optimal FRF with $L=0.7 \mathrm{H}$ and $R_{s}=180 \Omega,(\cdots)$ with no bolts on the upper bar of the frame, (--) with the upper line of piezoelectric patches which is short-circuited.

Fig. 7. Nevertheless, the evolution of the FRF is limited compared to the previous case involving the inductance modification. This observation is clear above $250 \mathrm{~Hz}$, where the short-circuits on the upper line of piezoelectric patches are almost ineffective. A reason is that the clamped-plate strain distribution induces a significant charge cancellation on those outer patches, especially when the wavelength approach their typical length. In the end, the proposed modification of a network boundary do not significantly alter the control performance.

\section{CONCLUSIONS}

A distributed control strategy is implemented by means of an interconnected array of piezoelectric patches. The interconnection is made through an electrical network analogous to the structure of interest. For a thin plate, we show that a finite difference method applied to the Kirchhoff-Love theory gives a discrete model that can be converted into its direct electrical analogue. The passive electrical network is coupled to an example clamped plate and tuned to minimize the velocity amplitude over a $50 \mathrm{~Hz}$ to $500 \mathrm{~Hz}$ frequency range. The resulting broadband vibration reduction validates the control strategy and the proposed electrical network. Then, structural and electrical modifications are introduced in order to quantify the performance loss due to an inaccurate tuning of the electromechanical system. The results obtained with the present setup give some guidelines concerning the robustness of a control solution based on analogous coupling. First, it is observed that a discrete network involving less than 5 unit cells per wavelength can still lead to a broadband vibration reduction above $20 \mathrm{~dB}$. Second, a $\pm 30 \%$ uniform variation of the network inductance induces a loss of $7 \mathrm{~dB}$, which maintains most of the control performance. Local defects are introduced by doubling the mass or the inductance of one unit cell. Boundaries are also modified by removing the bolts or short-circuiting the patches on the upper side of the plate. In any case, the FRF raises by less than $5 \mathrm{~dB}$ on the considered frequency range. It is thus shown that the proposed control involving the discrete analogue of a plate is relatively robust when considering an alteration of the mechanical structure or a detuning of the electrical network. This conclusion offers future prospects concerning the implementation of such control solutions into industrial applications.

\section{ACKNOWLEDGMENTS}

This work was funded by the French Ministry of National Education, Higher Education and Research through a three year scholarship for doctoral studies related to structural damping with piezoelectric devices. Moreover, the authors want to thank the Fulbright Program, which made possible a nine-month research visit at the Georgia Institute of Technology.

\section{REFERENCES}

[1] Spadoni, A., Ruzzene, M., and Cunefare, K. A., "Vibration and wave propagation control of plates with periodic arrays of shunted piezoelectric patches," J. Int. Mater. Sys. Struct. 20, 979-990 (2009).

[2] Casadei, F., Ruzzene, M., Dozio, L., and Cunefare, K. A., "Broadband vibration control through periodic arrays of resonant shunts: experimental investigation on plates," Smart Mater. Struct. 19, 015002 (2010).

[3] Chen, S., Wang, G., Wen, J., and Wen, X., "Wave propagation and attenuation in plates with periodic arrays of shunted piezo-patches," J. Sound Vib. 332, 1520-1532 (2013). 
[4] Wu, S. Y., "Method for multiple mode piezoelectric shunting with single pzt transducer for vibration control," J. Int. Mater. Sys. Struct. 9, 991-998 (1998).

[5] Airoldi, L. and Ruzzene, M., "Wave propagation control in beams through periodic multi-branch shunts," J. Int. Mater. Sys. Struct. 22, 1567-1579 (2011).

[6] Beck, B. S., Cunefare, K. A., and Collet, M., "Experimental assessment of negative impedance shunts for vibration suppression on a beam," in [Active and Passive Smart Structures and Integrated Systems 2008], Proc. SPIE 6928, 69281X-69281X-9 (2008).

[7] Casadei, F., Beck, B. S., Cunefare, K. A., and Ruzzene, M., "Vibration control of plates through hybrid configurations of periodic piezoelectric shunts," J. Int. Mater. Sys. Struct. 23, 1169-1177 (2012).

[8] Tateo, F., Collet, M., Ouisse, M., Ichchou, M. N., Cunefare, K. A., and Abbe, P., "Experimental characterization of a bi-dimensional array of negative capacitance piezo-patches for vibroacoustic control," J. Int. Mater. Sys. Struct. 26, 952-964 (2015).

[9] Vidoli, S. and Dell'Isola, F., "Vibration control in plates by uniformly distributed pzt actuators interconnected via electric networks," Eur. J. Mech. A Solid. 20, 435-456 (2001).

[10] Giorgio, I., Culla, A., and Vescovo, V. D., "Multimode vibration control using several piezoelectric transducers shunted with a multiterminal network," Arch. Appl. Mech. 79, 859-879 (2009).

[11] Bloch, A., "Electromechanical analogies and their use for the analysis of mechanical and electromechanical systems," J. Inst. Electr. Eng. 92, 157-169 (1945).

[12] Alessandroni, S., Dell'Isola, F., and Porfiri, M., "A revival of electric analogs for vibrating mechanical systems aimed to their efficient control by pzt actuators," Int. J. Solids Struct. 39, 5295-5324 (2002).

[13] MacNeal, R. H., The solution of partial differential equations by means of electrical networks, PhD thesis, California Institute of Technology (1949).

[14] Lossouarn, B., Aucejo, M., and Deü, J.-F., "Multimodal coupling of periodic lattices and application to rod vibration damping with a piezoelectric network," Smart Mater. Struct. 24, 045018 (2015).

[15] Lossouarn, B., Aucejo, M., and Deü, J.-F., "Multimodal vibration damping through a periodic array of piezoelectric patches connected to a passive network," in [Active and Passive Smart Structures and Integrated Systems 2015], Proc. SPIE 94311, 94311A-94311A-14 (2015).

[16] Porfiri, M., Dell'Isola, F., and Mascioli, F. M. F., "Circuit analog of a beam and its application to multimodal vibration damping, using piezoelectric transducers," Int. J. Circ. Theor. Appl. 32, 167-198 (2004).

[17] Lossouarn, B., Deü, J.-F., and Aucejo, M., "Multimodal vibration damping of a beam with a periodic array of piezoelectric patches connected to a passive electrical network," Smart Mater. Struct. 24, 115037 (2015).

[18] Alessandroni, S., Andreaus, U., DellIsola, F., and Porfiri, M., "A passive electric controller for multimodal vibrations of thin plates," Comput. Struct. 83, 1236-1250 (2005).

[19] Hagood, N. W. and von Flotow, A., "Damping of structural vibrations with piezoelectric materials and passive electrical networks," J. Sound Vib. 146, 243-268 (1991).

[20] Andreaus, U. and Porfiri, M., "Effect of electrical uncertainties on resonant piezoelectric shunting," J. Int. Mater. Sys. Struct. 18, 477-485 (2007).

[21] Berardengo, M., Cigada, A., Manzoni, S., and Vanali, M., "Vibration control by means of piezoelectric actuators shunted with lr impedances: performance and robustness analysis," Shock Vib. 2015 (2015).

[22] Gripp, J. A. B., Ges, L. C. S., Heuss, O., and Scinocca, F., "An adaptive piezoelectric vibration absorber enhanced by a negative capacitance applied to a shell structure," Smart Mater. Struct. 24, 125017 (2015).

[23] Thorp, O., Ruzzene, M., and Baz, A., "Attenuation and localization of wave propagation in rods with periodic shunted piezoelectric patches," Smart Mater. Struct. 10, 979 (2001).

[24] Rosi, G., Pouget, J., and Dell'Isola, F., "Control of sound radiation and transmission by a piezoelectric plate with an optimized resistive electrode," Eur. J. Mech. A Solid. 29, 859-870 (2010).

[25] Lossouarn, B., Cunefare, K. A., Aucejo, M., and Deü, J.-F., "Multimodal damping of a plate with a passive piezoelectric network," Proc. IMAC-XXXIV (2016). 